\title{
Diversity of the Cyanoprokaryota of the area of settlement Pyramiden, West Spitsbergen Island, Spitsbergen archipelago
}

\author{
Denis Davydov \\ Polar Alpine Botanical Garden Institute Kola SC RAS, 184256, Botanical Garden, Kirovsk, Murmansk Region, Russia; \\ E-mail: d_disa@mail.ru
}

\begin{abstract}
The diversity and ecological distribution of cyanoprokaryotes in the area of settlement Pyramiden of West Spitsbergen Island, Spitsbergen (Svalbard) archipelago, were studied during the summer seasons 2008 and 2013. A total of 73 taxa were observed in various habitats of investigated area. The highest number of species was found on wet seepages on the slopes (33 species), in slow tundra streams (26 species) and wet soils (21 species). Nostoc commune and Calothrix parietina dominate in most parts of the habitats.
\end{abstract}

\section{INTRODUCTION}

Cyanoprokaryota (Cyanophyta, Cyanobacteria) comprise prominent and essential autotrophic component of polar biota. They are intense producers of organic biomass during the polar summer season (Komárek et al., 2012). Cyanoprokaryota are widespread in all polar environments. They play a key role in Arctic ecosystems as diazotrophs.

Study of the cyanoprokaryotes of Spitsbergen archipelago began in the 19th century (Skulberg, 1996). There are numerous publications concerning the diversity of the freshwater and terrestrial cyanoprokaryota of Spitsbergen archipelago (Willen, 1980; Matuła, 1982; Plichta \& Luścińska, 1988; Matuła \& Swies, 1989; Perminova, 1990; Oleksowicz \& Luścińska, 1992; Davydov, 2005, 2008, 2010, 2011, 2013; Kaštovská et al., 2005; Turicchia et al., 2005; Komárek et al., 2006, 2012; Matuła et al., 2007; Kim et al., 2008, 2011; Richter et al., 2009).

The present study adds new information on the freshwater and terrestrial cyanoprokaryota on the Spitsbergen archipelago. The data were collected during July-August 2008 and 2013.

\section{MATERIAL AND METHODS}

\section{Study area}

The investigated area is located in the central part of Spitsbergen, in the eastern part of the Dickson Land, on the western shore of Billefjorden. The relief of this part of Spitsbergen is sharply dissected and characterized by signifi- cant elevations. Area is surrounded by mountains which reach from 265 up to $935 \mathrm{~m}$ a.s.1. The investigated territory is situated within the field of rocks of the Paleozoic (541 to 252 million years ago).

The rocks of the Lower Carboniferous (358,9 to 298,9 million years ago) are widespread. They are presented by conglomerates, sandstones and limestones with local occurrence of coal. Also in the area there are Devonian (419.2 to 358.9 million years ago) rocks, composed of sandstone, siltstone, quartzitic sandstone and mudstone.

The hydrological system of the area is quite extensive. It is presented by the river Mimer and its tributaries, which occupy the central part of the valley. River supply is provided by the melting glaciers and to a lesser extent by precipitation. Flooding takes place in June and July, mean water is in August.

Soil profile is shallow and typical for the high Arctic. Soil cover is patterned; most part of soils has developed on topography that has been shaped by cryoplanation, on materials disturbed by sloping processes and erosion.

Climate of the area is determined by the influence of several ocean currents, one of them, branch of the Gulf Stream, is of particular importance. It is responsible for high air humidity and precipitation. July is the warmest month with average temperature $+6.8^{\circ} \mathrm{C}$ (Petchurov, 1983; more in detail Láska et al., 2012).

\section{Methods}

Samples were collected during 25 July-3 August, 2008 and 1-10 August, 2013 in the 
area covering Mimerdalen, Tordalen valleys, Planteryggen, Reuterskiöldfjellet, Pyramiden, Svenbrehøgda mountain slopes (Fig. 1, Table 1). In total, 243 samples were collected. Least number samples were collected under the bird colonies (6) and in fast streams (5), and the maximum in the slow streams (26) and seepages
(33). The species of cyanoprokaryota were identified, measured and photographed using the optical microscope AxioScope A1 (Zeiss $C$ ). The presence or absence of species on the samples were recorded; the abundance of species was not considered. For species identification, essential monographs were used (Geitler, 1932;

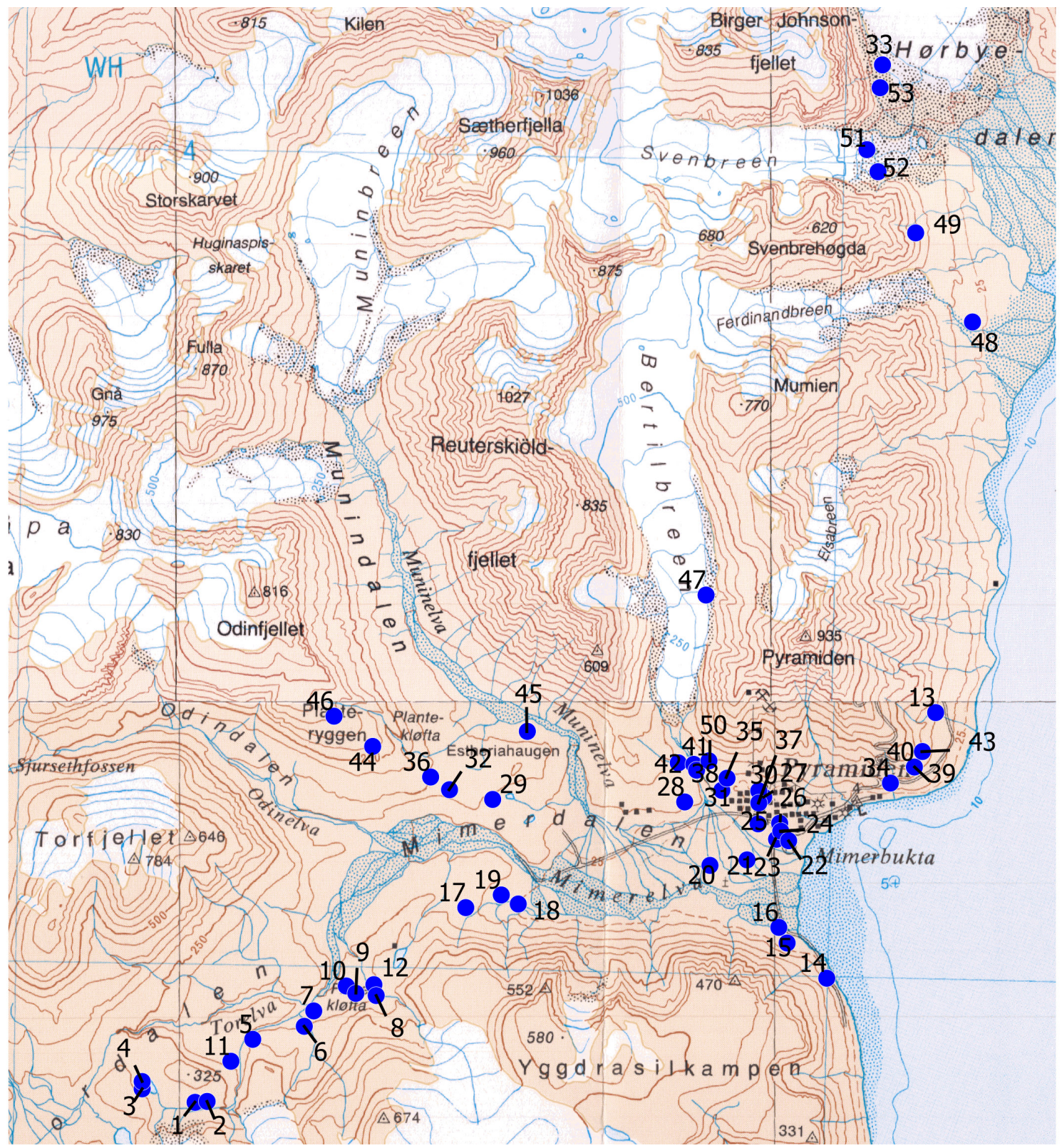

Fig. 1. Map of the area nearby settlement Pyramiden of West Spitsbergen Island, Spitsbergen archipelago; dots are sample plots enumerated. Description of plots see in Table 1. 
Table 1. Description of studied localities

\begin{tabular}{|c|c|c|c|c|}
\hline $\begin{array}{l}\text { No. of } \\
\text { locality }\end{array}$ & Latitude N & Longitude $\mathrm{E}$ & $\begin{array}{l}\text { Elevation } \\
\text { (m a.s.l.) }\end{array}$ & Description of localities \\
\hline 1 & 78.6224 & 16.0113 & 267 & $\begin{array}{l}\text { Valley Tordalen. Mountain Jotunrabbane, slope of NW exposure. } \\
\text { Fast stream flowing with glacier Jotunfonna. Flooded gravelly } \\
\text { plain. On the soil. }\end{array}$ \\
\hline 2 & 78.6225 & 16.0180 & 267 & $\begin{array}{l}\text { Valley Tordalen. Mountain Jotunrabbane, slope of NW exposure. } \\
\text { Fast stream flowing with glacier Jotunfonna. On the rock in wa- } \\
\text { terfall. }\end{array}$ \\
\hline 3 & 78.6238 & 15.9816 & 279 & Valley Tordalen. Coast of the lake. \\
\hline 4 & 78.6247 & 15.9816 & 285 & Valley Tordalen. Coast of the lake. Seepage. On the flooded soil. \\
\hline 5 & 78.6293 & 16.0436 & 219 & Mountain Yggdrasilkampen, valley Fiskekløfta. On the soil. \\
\hline 6 & 78.6307 & 16.0725 & 168 & $\begin{array}{l}\text { Valley Mimerdalen. Mimer river. Wet rock next waterfall. On the } \\
\text { sand. }\end{array}$ \\
\hline 7 & 78.6333 & 16.0778 & 156 & $\begin{array}{l}\text { Valley Mimerdalen. Mountain Yggdrasilkampen, slope of NNW } \\
\text { exposure. Stream. Mats at the bottom on the sand. }\end{array}$ \\
\hline 8 & 78.6342 & 16.1128 & 180 & $\begin{array}{l}\text { Valley Mimerdalen. Mountain Yggdrasilkampen, slope of NNW } \\
\text { exposure. Snow-fed stream, and seepage on the slope. On the sand } \\
\text { or soil. }\end{array}$ \\
\hline 9 & 78.6344 & 16.1013 & 150 & $\begin{array}{l}\text { Valley Tordalen. Mountain Jotunrabbane, slope of NW exposure. } \\
\text { Wet moss tundra. On the sand between small stones. }\end{array}$ \\
\hline 10 & 78.6352 & 16.0960 & 138 & $\begin{array}{l}\text { Mountain Yggdrasilkampen, valley Fiskekløfta. At the bottom of } \\
\text { the shallow pool. }\end{array}$ \\
\hline 11 & 78.6269 & 16.0313 & 175 & Valley Tordalen. Stream flowing with glacier Jotunfonna. \\
\hline 12 & 78.6353 & 16.1114 & 180 & $\begin{array}{l}\text { Valley Mimerdalen. Mountain Yggdrasilkampen, slope of SE ex- } \\
\text { posure. Bank of the snow-fed stream. On the soil. }\end{array}$ \\
\hline 13 & 78.6653 & 16.4263 & 5 & $\begin{array}{l}\text { Mountain Pyramiden slope of ESE exposure. Local depression, } \\
\text { Coast of the stream. On the soil. }\end{array}$ \\
\hline 14 & 78.6361 & 16.3652 & 18 & $\begin{array}{l}\text { Coast of the see. Basis of the mountain Yggdrasilkampen. Fast } \\
\text { stream. On the sand. }\end{array}$ \\
\hline 15 & 78.6400 & 16.3430 & 40 & $\begin{array}{l}\text { Valley Mimerdalen. Mountain Yggdrasilkampen. Slope of NNW } \\
\text { exposure. On the soil. }\end{array}$ \\
\hline 16 & 78.6417 & 16.3384 & 2 & $\begin{array}{l}\text { Valley Mimerdalen. Right coast of the river. Basis of the mountain } \\
\text { Yggdrasilkampen. Coast of the stream. On the clay. }\end{array}$ \\
\hline 17 & 78.6439 & 16.1630 & 100 & $\begin{array}{l}\text { Mountain Yggdrasilkampen. Slope of NNW exposure. Coast of } \\
\text { the stream. At the bottom of the shallow pool. }\end{array}$ \\
\hline 18 & 78.6443 & 16.1924 & 105 & $\begin{array}{l}\text { Mountain Yggdrasilkampen. Slope of NNW exposure. Flooded } \\
\text { moss tundra, between tussocks. On the mosses. }\end{array}$ \\
\hline 19 & 78.6453 & 16.1806 & 105 & $\begin{array}{l}\text { Mountain Yggdrasilkampen. Slope of NNW exposure. Small } \\
\text { stream. On the stones. }\end{array}$ \\
\hline 20 & 78.6485 & 16.2998 & 13 & Valley Mimerdalen. Wet moss tundra. On the soil. \\
\hline 21 & 78.6491 & 16.3206 & 6 & Valley Mimerdalen. On the alluvium (sand). \\
\hline 22 & 78.6512 & 16.3439 & 9 & $\begin{array}{l}\text { Territory of settlement. Coastal of the see. See marsh. Puccinellia } \\
\text { phryganodes and Stellaria humifusa community. Dark braun mats. } \\
\text { On the soil. }\end{array}$ \\
\hline 23 & 78.6514 & 16.3371 & 2 & Coastal of the see. Wet meadow. On the sand. \\
\hline 24 & 78.6523 & 16.3395 & 9 & $\begin{array}{l}\text { Territory of settlement. Wetland. Eriophorum scheuchzeri commu- } \\
\text { nity. On the soil. }\end{array}$ \\
\hline
\end{tabular}


Table 1 (continued)

\begin{tabular}{|c|c|c|c|c|}
\hline $\begin{array}{l}\text { No. of } \\
\text { locality }\end{array}$ & Latitude $\mathrm{N}$ & Longitude $\mathrm{E}$ & $\begin{array}{l}\begin{array}{c}\text { Elevation } \\
\text { (m a.s.l.) }\end{array} \\
\end{array}$ & Description of localities \\
\hline 25 & 78.6531 & 16.3268 & 103 & $\begin{array}{l}\text { Territory of settlement. Anthropogenous meadow about cow- } \\
\text { house. On the soil. }\end{array}$ \\
\hline 26 & 78.6532 & 16.3389 & 9 & Territory of settlement. At the bottom of the shallow pool. \\
\hline 27 & 78.6560 & 16.3299 & 9 & Territory of settlement. Under bird colony. On the soil. \\
\hline 28 & 78.6555 & 16.2856 & 50 & $\begin{array}{l}\text { Mountain Reuterskiöldfjellet. Mountain terrace, local depression. } \\
\text { Salix and moss comunity. Seepage. On the soil. }\end{array}$ \\
\hline 29 & 78.6558 & 16.1780 & 98 & $\begin{array}{l}\text { Mountain Planteryggen. Coastal of the lake. Flooded moss tun- } \\
\text { dra. On the mosses. }\end{array}$ \\
\hline 30 & 78.6567 & 16.3271 & 25 & $\begin{array}{l}\text { Territory of settlement. Anthropogenous meadow between two } \\
\text { roads On the soil. }\end{array}$ \\
\hline 31 & 78.6568 & 16.3064 & 16 & $\begin{array}{l}\text { Territory of settlement. The former stadium. Side slopes. On the } \\
\text { soil. }\end{array}$ \\
\hline 32 & 78,6568 & 16.1539 & 80 & $\begin{array}{l}\text { Mountain Planteryggen. Slope of N exposure. Rocks. Local de- } \\
\text { pression. On the soil. }\end{array}$ \\
\hline 33 & 78.7365 & 16.3965 & 100 & $\begin{array}{l}\text { Petuniabukta bay. Mountain Birger Johnsonfjellet. Slope of E ex- } \\
\text { posure. Valley Hørbyedalen. Bog with tussocks. On the clay. }\end{array}$ \\
\hline 34 & 78.6576 & 16.4009 & 42 & $\begin{array}{l}\text { Mountain Pyramiden. Slope of S exposure. Fragmented Dryas oc- } \\
\text { topetala community. On the soil. }\end{array}$ \\
\hline 35 & 78.6581 & 16.3093 & 36 & $\begin{array}{l}\text { Mountain Pyramiden, submontane stream forming the seepage. } \\
\text { Outside the main stream at a relatively dry areas. }\end{array}$ \\
\hline 36 & 78.6583 & 16.1432 & & $\begin{array}{l}\text { Slope of the hill of } S \text { exposure. Snow-fed stream. Mats on the } \\
\text { rock. }\end{array}$ \\
\hline 37 & 78.6554 & 16.3271 & 48 & Territory of settlement. Under bird colony. On the soil. \\
\hline 38 & 78.6590 & 16.2923 & 71 & Mountain Pyramiden. Dryas octopetala community. On the soil. \\
\hline 39 & 78.6594 & 16.4144 & & $\begin{array}{l}\text { Mountain Pyramiden. Slope of SE exposure. Lichens tundra and } \\
\text { Cassiopa tetragona, Dryas octopetala and Silene acaulis. On small } \\
\text { hillocks (soil). }\end{array}$ \\
\hline 40 & 78.6594 & 16.4144 & & $\begin{array}{l}\text { Mountain Pyramiden. Slope of SE exposure. Lichens tundra and } \\
\text { Cassiopa tetragona, Dryas octopetala and Silene acaulis. On small } \\
\text { hillocks (soil). }\end{array}$ \\
\hline 41 & 78.6596 & 16.2907 & 72 & $\begin{array}{l}\text { Mountain Pyramiden. Slope of S exposure. Cassiope tetragona } \\
\text { community. On the soil. }\end{array}$ \\
\hline 42 & 78.6598 & 16.2820 & 53 & $\begin{array}{l}\text { Mountain Reuterskiöldfjellet. Slope of } S \text { exposure. Stream on the } \\
\text { cleft. On the mosses. }\end{array}$ \\
\hline 43 & 78.6611 & 16.4188 & 88 & $\begin{array}{l}\text { Mountain Pyramiden. Slope of SSE exposure. Mountain terrace, } \\
\text { local depression. On the soil. }\end{array}$ \\
\hline 44 & 78.6616 & 16.1108 & 259 & $\begin{array}{l}\text { Mountain Odinfellet. Plantekløfta valley. Slope of SSE exposure. } \\
\text { Salix polaris, Dryas octopetala and mosses community. On the } \\
\text { mosses. }\end{array}$ \\
\hline 45 & 78.6633 & 16.1975 & 98 & $\begin{array}{l}\text { Valley Munindalen. Slope of N exposure. Stream. Mats on the } \\
\text { rock. }\end{array}$ \\
\hline 46 & 78.6650 & 16.0891 & 369 & $\begin{array}{l}\text { Mountain Planteryggen. Slope of SSE exposure. Snow-fed stream } \\
\text { forming the seepage. }\end{array}$ \\
\hline 47 & 78.6783 & 16.2976 & 250 & $\begin{array}{l}\text { Valley Bertilbreen. Mountain Pyramiden. Slope of W exposure. } \\
\text { On the soil between stone. }\end{array}$ \\
\hline
\end{tabular}


Table 1 (continued)

\begin{tabular}{|c|c|c|c|c|}
\hline $\begin{array}{l}\text { No. of } \\
\text { locality }\end{array}$ & Latitude $\mathrm{N}$ & Longitude E & $\begin{array}{r}\text { Elevation } \\
\text { (m a.s.l.) }\end{array}$ & Description of localities \\
\hline 48 & 78.7083 & 16.4469 & 29 & $\begin{array}{l}\text { Petuniabukta bay. River flowing from glacier Ferdinandbreen. } \\
\text { Mountain Mumien. Mountain terrace. Wet moss tundra. On the } \\
\text { soil. }\end{array}$ \\
\hline 49 & 78.7181 & 16.4151 & 70 & $\begin{array}{l}\text { Petuniabukta bay. Mountain Svenbrehøgda. Rock } 3-5 \mathrm{~m} \text { in height. } \\
\text { In the shade on the rock. }\end{array}$ \\
\hline 50 & 78.6600 & 16.2992 & 56 & $\begin{array}{l}\text { Mountain Pyramiden. Slope of S exposure. Stream. Oxyria digyna } \\
\text { and mosses community. }\end{array}$ \\
\hline 51 & 78.7272 & 16.3877 & 104 & $\begin{array}{l}\text { Petuniabukta bay. Valley of river flowing down from glacier Sven- } \\
\text { breen. Under rocks. On the soil. }\end{array}$ \\
\hline 52 & 78.7248 & 16.3940 & 50 & $\begin{array}{l}\text { Petuniabukta bay. Base of the mountain Svenbrehøgda. Slope of } \\
\text { N exposure. Rock 3-5 m height. The wall of SE exposure. On the } \\
\text { rock. }\end{array}$ \\
\hline 53 & 78.7340 & 16.3952 & 100 & $\begin{array}{l}\text { Petuniabukta bay. Mountain Birger Johnsonfjellet. Slope of E ex- } \\
\text { posure. Small stream. }\end{array}$ \\
\hline
\end{tabular}

Hollerbach et al., 1953; Komárek \& Anagnostidis, 1998, 2005; Komarek, 2013). Information on habitats and descriptions of localities were submitted into the CYANOpro database (http:// kpabg.ru/cyanopro/) (Melechin et al., 2013).

In this study we have attempted to focus on the microhabitat as the primary environment for the distribution of cyanoprokaryotes, with the goal of defining niches for individual species. The term "microhabitat" refers to a small section of the habitat that is spatially homogeneous in both biotic and abiotic factors, i.e., a section of rock, gravelly, soils and other substrata with relatively the same moisture, illumination, etc. For each sample environmental parameters were considered and the habitat type determined (Table 2). To estimate the breadths of ecological niches, the Stephenson's formula (1988) was used: $N B=1 /\left(n \sum P_{i j}{ }^{2}\right)$, where NB - niche breadth, $n$ - the number of habitat types, $P_{i j}$ - the proportion of species $i$ in the habitat type $j$, which is calculated as the ratio of the number of samples $\mathrm{i}^{\text {th }}$ species in the $\mathrm{j}^{\text {th }}$ type to the total number of samples of this species. Thus, for determining niche breadth, the number of habitat types where the taxon was found was taken into account. Values for NB range from 0 to 1 .

Floristic similarity was found using the Sørensen index (KS) (recommended, for example, by Wolda, 1981) (weighted pair-group method using arithmetic averaging) in the program module GRAPHS (Novakovskij, 2004): KS = 2a/
$(2 a+b+c)$, where $a-$ number of species common to both sets, $b$ - number of species unique to the first set, $\mathrm{c}$ - number of species unique to the set.

\section{RESULTS AND DISCUSSION}

A total of 73 cyanobacterial taxa were identified in the habitats of investigated area (Table 2). Matuła et al. (2007) identified 100 species of Cyanoprokaryota in Revelva valley in the Hornsund Bay. More than 80 morphospecies were registered in Petunia Bay (Komárek et al., 2012). Eleven species are first time records for Spitsbergen flora: Anabaena inaequalis, Calothrix aeruginosa, Chroococcus spelaeus, C. subnudus, Gloeocapsa rupicola, G. violascea, Gloeothece palea, Leptolyngbya bijugata, Lyngbya martensiana, Rivularia coadunate, Trichocoleus sociatus.

Nostoc commune (58 observations), Calothrix parietina (11 observations), Microcoleus autumnalis (9 observations) were the most common species in the investigated samples (Table 2).

Cyanoprokaryotes communities are developed mainly in places with high humidity. The highest number of species was found on wet seepages on the slopes (33 species), in slow tundra streams (26 species) and on the wet soils (21 species, table 3 ). The most common species on the wet slope seepages were Aphanocapsa cf. grevillei and Aphanothece saxicola. Chroococcus cohaerens and Gloeocapsa alpina were found in three habitats: on seepages, wet soils and on 
Table 2. List of cyanoprokaryote taxa found in different habitats ${ }^{1}$

\begin{tabular}{|c|c|c|c|c|c|c|c|c|c|c|c|c|c|c|}
\hline \multirow[t]{2}{*}{$\overline{\text { Species }}$} & \multicolumn{12}{|c|}{ Habitats } & \multirow[t]{2}{*}{ NS } & \multirow[t]{2}{*}{ NB } \\
\hline & FS & SS & WT & $\mathrm{P}$ & $S$ & WS & WR & SM & $\mathrm{CL}$ & $\mathrm{A}$ & $\mathrm{M}$ & $\mathrm{BC}$ & & \\
\hline $\begin{array}{l}\text { Anabaena inaequalis (Kütz.) } \\
\text { Born. et Flah. }{ }^{2}\end{array}$ & - & - & - & - & - & - & - & 22 & - & - & 25 & - & 6 & 0,12 \\
\hline $\begin{array}{l}\text { Aphanocapsa muscicola } \\
\text { (Menegh.) Wille }\end{array}$ & - & - & 24 & - & 28 & 8,32 & - & 22 & - & - & - & - & 4 & 0,33 \\
\hline Aphanocapsa parietina Näg. & - & 18 & - & - & - & - & 49 & 23 & - & - & - & - & 3 & 0,25 \\
\hline $\begin{array}{l}\text { Aphanocapsa cf. fusco-lutea } \\
\text { Hansg. }\end{array}$ & - & - & - & - & - & 32,40 & - & - & - & - & - & - & 2 & 0,08 \\
\hline $\begin{array}{l}\text { Aphanocapsa cf. grevillei } \\
\text { (Berk.) Rabenh. }\end{array}$ & - & - & - & - & 35 & - & - & - & - & - & - & - & 1 & $\mathbf{0 , 0 8}$ \\
\hline Aphanocapsa sp. & - & - & - & 10 & $\begin{array}{l}28, \\
46\end{array}$ & 1 & 52 & - & 3 & - & - & - & 5 & 0,08 \\
\hline $\begin{array}{l}\text { Aphanothece caldariorum } \\
\text { Richter }\end{array}$ & - & - & - & - & - & 32 & - & - & - & - & - & - & 2 & $\mathbf{0 , 0 8}$ \\
\hline $\begin{array}{l}\text { Aphanothece castagnei (Bréb.) } \\
\text { Rabenh. }\end{array}$ & - & - & 24 & - & 28 & 48 & - & - & - & - & - & - & 3 & 0,17 \\
\hline Aphanothece saxicola Näg. & - & - & - & - & 28 & - & - & - & - & - & - & - & 2 & 0,08 \\
\hline $\begin{array}{l}\text { Calothrix aeruginosa } \\
\text { Voronich. }^{2}\end{array}$ & - & 42 & - & - & - & - & - & - & - & - & - & - & 1 & 0,08 \\
\hline $\begin{array}{l}\text { Calothrix breviarticulata W. } \\
\text { West et G.S. West }\end{array}$ & - & 53 & - & - & - & - & - & - & - & - & - & - & 1 & 0,08 \\
\hline $\begin{array}{l}\text { Calothrix parietina Thur. ex } \\
\text { Born. et Flah. }\end{array}$ & - & $\begin{array}{l}18 \\
42\end{array}$ & - & 10 & $\begin{array}{l}28, \\
46\end{array}$ & $\begin{array}{l}1,5 \\
48\end{array}$ & $\begin{array}{l}49 \\
52\end{array}$ & - & 3 & 21 & - & - & 12 & 0,48 \\
\hline $\begin{array}{l}\text { Chroococcus cohaerens (Bréb.) } \\
\text { Näg. }\end{array}$ & - & - & - & - & $\begin{array}{l}28, \\
46\end{array}$ & 12 & 49 & - & - & - & - & - & 4 & 0,22 \\
\hline
\end{tabular}

$\begin{array}{lllllllllllllllllll}\text { Chroococcus helveticus Näg. } & - & 36 & - & - & - & - & - & - & - & - & - & - & \mathbf{0 , 0 8}\end{array}$

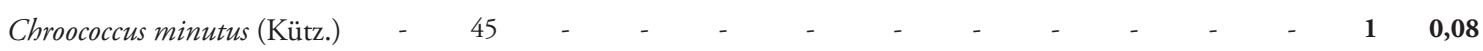

Näg.

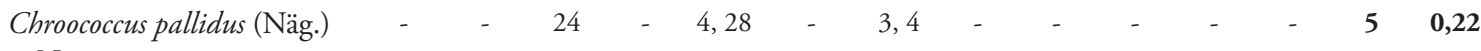

Näg.

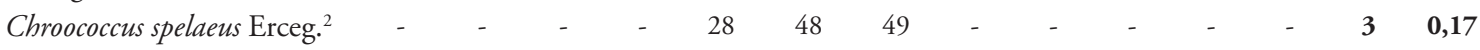

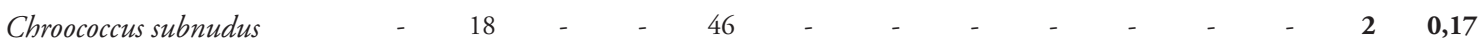

(Hansg.) Cronb. et

Komárek $^{2}$

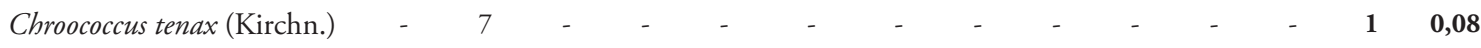

Hieron.

Chroococcus varius A. Braun - $\quad \begin{array}{llllllllllllll}36 & - & 10 & 28 & 32,39 & 3 & - & 3 & - & - & - & \mathbf{8} & \mathbf{0 , 3 8}\end{array}$

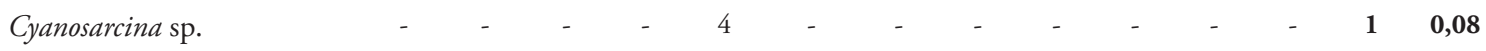

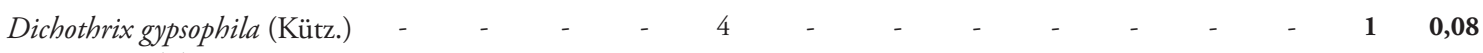

Born. et Flah.

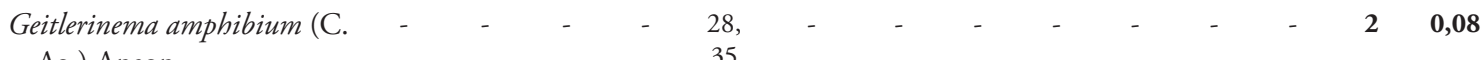

Ag.) Anagn.

Gloeocapsa alpina (Näg.)

Brand

$\begin{array}{llllllll}4 & 32,39, & 3 & - & 3 & - & - & \end{array} \quad \mathbf{0}, 22$

Gloeocapsa decorticans (A.

Braun) Richt.

10

40

$2 \quad \mathbf{0 , 1 7}$ 
Table 2 (continued)

\begin{tabular}{|c|c|c|c|c|c|c|c|c|c|c|c|c|c|c|}
\hline \multirow[t]{2}{*}{ Species } & \multicolumn{12}{|c|}{ Habitats } & \multirow[t]{2}{*}{ NS } & \multirow[t]{2}{*}{ NB } \\
\hline & FS & SS & WT & $\mathrm{P}$ & $S$ & WS & WR & SM & $\mathrm{CL}$ & A & $\mathrm{M}$ & $\mathrm{BC}$ & & \\
\hline Gloeocapsa kuetzingiana Näg. & - & - & - & - & 28 & $\begin{array}{c}15,38 \\
39\end{array}$ & - & - & - & - & - & - & 4 & 0,14 \\
\hline Gloeocapsa rupicola Kütz. ${ }^{2}$ & - & 42 & - & - & - & - & - & - & - & - & - & - & 1 & 0,08 \\
\hline $\begin{array}{l}\text { Gloeocapsa sanguinea (C. Ag.) } \\
\text { Kütz. }\end{array}$ & - & - & - & - & 46 & 5 & 49 & - & - & - & - & - & 3 & 0,25 \\
\hline $\begin{array}{l}\text { Gloeocapsa violascea (Corda) } \\
\text { Rabenh. }^{2}\end{array}$ & - & 7,53 & - & - & $\begin{array}{l}28 \\
46\end{array}$ & $\begin{array}{c}1,12 \\
15,32 \\
48\end{array}$ & 49 & - & - & - & - & - & 8 & 0,27 \\
\hline Gloeothece confluens Näg. & - & 17 & 9 & - & - & $\begin{array}{c}8,15 \\
43\end{array}$ & 49 & - & - & 21 & - & - & 7 & 0,22 \\
\hline $\begin{array}{l}\text { Gloeothece cyanochroa } \\
\text { Komárek }\end{array}$ & - & - & - & 10 & 28 & - & - & - & - & - & - & - & 2 & 0,17 \\
\hline $\begin{array}{l}\text { Gloeothece palea (Kütz.) } \\
\text { Rabenh. }^{2}\end{array}$ & - & - & - & - & 4 & 8 & - & - & - & - & - & - & 2 & 0,17 \\
\hline $\begin{array}{l}\text { Gloeothece cf. violacea Ra- } \\
\text { benh. }\end{array}$ & - & - & - & - & 46 & - & - & - & - & - & - & - & 1 & 0,08 \\
\hline Gloeothece sp. & - & - & - & - & 46 & - & - & - & - & - & - & - & 1 & 0,08 \\
\hline $\begin{array}{l}\text { Leptolyngbya bijugata (Kon- } \\
\text { giss.) Anagn. et Komárek }\end{array}$ & - & - & - & - & 4 & - & - & - & - & - & - & - & 1 & 0,08 \\
\hline $\begin{array}{l}\text { Leptolyngbya gracillima (Zopf } \\
\text { ex Hansg.) Anagn. et } \\
\text { Komárek }\end{array}$ & - & - & - & - & - & 43 & - & - & - & - & - & - & 1 & 0,08 \\
\hline Leptolyngbya cf. gracillima & - & - & - & - & 28 & - & - & - & - & - & - & - & 1 & 0,08 \\
\hline Leptolyngbya sp. & - & - & - & 10 & - & - & - & - & - & - & - & - & 1 & 0,08 \\
\hline $\begin{array}{l}\text { Lyngbya martensiana } \\
\text { Menegh. ex Gom. }\end{array}$ & - & - & - & 26 & - & - & - & - & - & - & - & - & 1 & 0,08 \\
\hline $\begin{array}{l}\text { Microcoleus amoenus (Kütz. } \\
\text { ex Gom.) Strunecky et al. }\end{array}$ & - & - & - & 17 & - & - & - & - & - & - & - & - & 1 & 0,08 \\
\hline $\begin{array}{l}\text { Microcoleus autumnalis (Trev. } \\
\text { ex Gom.) Strunecky et al. }\end{array}$ & - & $\begin{array}{l}36, \\
53\end{array}$ & 24 & $\begin{array}{l}10, \\
26\end{array}$ & 35 & - & - & - & - & - & 25 & $\begin{array}{l}27 \\
37\end{array}$ & 9 & 0,22 \\
\hline $\begin{array}{l}\text { Microcoleus vaginatus Gom. } \\
\text { ex Gom. }\end{array}$ & - & $\begin{array}{l}42 \\
50 \\
53\end{array}$ & 9 & - & 46 & $\begin{array}{l}1,8 \\
34,48\end{array}$ & 49 & - & - & - & - & - & 7 & 0,27 \\
\hline $\begin{array}{l}\text { Nostoc commune Vauch. ex } \\
\text { Born. et Flah. }\end{array}$ & + & $\begin{array}{c}7,13 \\
17- \\
19 \\
36 \\
45 \\
50\end{array}$ & $\begin{array}{l}9, \\
24, \\
29, \\
33, \\
39 \\
44\end{array}$ & 10 & $\begin{array}{l}4, \\
28, \\
35, \\
30, \\
46\end{array}$ & $\begin{array}{c}1,5,8, \\
12,15, \\
20,28, \\
31,32, \\
34,38, \\
41,43, \\
47,48, \\
51\end{array}$ & $\begin{array}{l}49 \\
52\end{array}$ & $\begin{array}{l}22 \\
23\end{array}$ & - & $\begin{array}{l}16 \\
21\end{array}$ & 25 & - & 58 & 0,27 \\
\hline
\end{tabular}

Nostoc microscopicum Carm. ex Born. et Flah.

Nostoc minutum Desmaz. ex Born. et Flah.

Nostoc sp. 
Table 2 (continued)

\begin{tabular}{|c|c|c|c|c|c|c|c|c|c|c|c|c|c|c|}
\hline \multirow[t]{2}{*}{ Species } & \multicolumn{12}{|c|}{ Habitats } & \multirow[t]{2}{*}{ NS } & \multirow[t]{2}{*}{ NB } \\
\hline & $\mathrm{FS}$ & SS & WT & $\mathrm{P}$ & $S$ & WS & WR & SM & $\mathrm{CL}$ & $\mathrm{A}$ & $\mathrm{M}$ & $\mathrm{BC}$ & & \\
\hline $\begin{array}{l}\text { Oscillatoria sancta Kütz. ex } \\
\text { Gom. }\end{array}$ & - & 42 & - & - & - & - & - & - & - & - & - & - & 2 & 0,08 \\
\hline $\begin{array}{l}\text { Oscillatoria simplicissima } \\
\text { Gom. }\end{array}$ & - & - & - & 10 & - & - & - & - & - & - & - & - & 1 & 0,08 \\
\hline $\begin{array}{l}\text { Oscillatoria tenuis C. Ag. ex } \\
\text { Gom. }\end{array}$ & - & $\begin{array}{c}6,19 \\
45\end{array}$ & 33 & - & 46 & - & - & - & - & - & - & - & 5 & 0,19 \\
\hline $\begin{array}{l}\text { Oscillatoria cf. limosa C. Ag. } \\
\text { ex Gom. }\end{array}$ & - & 36 & - & - & - & - & - & - & - & - & - & - & 1 & 0,08 \\
\hline Oscillatoria sp. & - & 8,36 & - & - & - & - & - & - & - & - & - & - & 2 & 0,08 \\
\hline $\begin{array}{l}\text { Petalonema incrustans [Kütz.] } \\
\text { Komárek }\end{array}$ & - & - & - & - & - & 32 & - & - & - & - & - & - & 2 & 0,08 \\
\hline $\begin{array}{l}\text { Phormidium kuetzingianum } \\
\text { (Kirchn.) Anagn. et } \\
\text { Komárek }\end{array}$ & - & $\begin{array}{l}17 \\
45\end{array}$ & - & - & 46 & - & - & - & - & - & - & - & 4 & 0,13 \\
\hline Phormidium uncinatum & - & 42 & - & 10 & - & - & - & - & - & - & - & - & 4 & 0,22 \\
\hline
\end{tabular}

Gom. ex Gom.

Phormidium cf. calcareum

Kütz.

Phormidium sp.

Pseudanabaena frigida

(Fritsch) Anagn.

Rhabdoderma irregulare

(Naum.) Geitl.

Rivularia coadunata (Somm.)

Foslie $^{2}$

18 ,

19

Schizothrix arenaria (Berk.)

36

Gom.

Schizothrix facilis (Skuja)

Anagn.

Schizothrix tinctoria (C. Ag.)

Gom.

Schizothrix sp.

Scytonema myochrous

(Dillwyn) C. Ag. ex Born.

et Flah.

Scytonema ocellatum Lyngb. ex Born. et Flah.

Spirulina tenerrima Kütz. ex Gom.

Stigonema ocellatum (Dillw.) 
Table 2 (continued)

\begin{tabular}{|c|c|c|c|c|c|c|c|c|c|c|c|c|c|c|}
\hline \multirow[t]{2}{*}{ Species } & \multicolumn{12}{|c|}{ Habitats } & \multirow[t]{2}{*}{ NS } & \multirow[t]{2}{*}{ NB } \\
\hline & FS & SS & WT & $\mathrm{P}$ & $S$ & WS & WR & SM & $\mathrm{CL}$ & A & $M$ & $\mathrm{BC}$ & & \\
\hline Tolypothrix saviezii Kossinsk. & - & - & - & - & 28 & - & - & - & - & - & - & - & 1 & 0,08 \\
\hline $\begin{array}{l}\text { Tolypothrix tenuis Kütz. ex } \\
\text { Born. et Flah. }\end{array}$ & 6 & - & - & - & - & - & - & - & - & - & - & - & 1 & 0,08 \\
\hline Tolypothrix sp. & - & - & - & - & $\begin{array}{l}13, \\
46\end{array}$ & - & - & - & - & - & - & - & 2 & 0,08 \\
\hline $\begin{array}{l}\text { Trichocoleus sociatus (W. West } \\
\text { et G. S. West) Anagn. }{ }^{2}\end{array}$ & - & - & - & 10 & - & - & - & 23 & - & 21 & - & - & 3 & 0,25 \\
\hline
\end{tabular}

${ }^{1}$ - Numbers within the columns correspond to the locality numbers in Table $1 ;^{2}$ - first records for Spitsbergen flora. Habitats: FS - fast running glacial streams and waterfalls; SS - slow running tundra streams; WT - wet moss tundra; P - tundra pools; S - seepages; WS - wetted soils; WR - wet and dripping rocks; SM - coastal to the sea, sea marsh; CL - coast of the lake; A - alluvium on the riverside; $\mathrm{M}$ - meadow about cow-house, on the soil; BC - under bird colony, on the soil. NS - number of samples; NB - values for niche breadth.

the rocks. The most common taxa on the soil were Calothrix parietina, Microcoleus vaginatus, Nostoc commune. Only on wet soils were found Aphanocapsa cf. fusco-lutea and Stigonema ocellatum. At the bottom of the slow running streams cyanobacterial communities were dominated by Phormidium uncinatum. A total of 26 species of cyanoprokaryota were found on the slow running streams.

Most similar was the species composition of the meadow nearby cow-house and on the soils under the bird colony, wet tundra habitat were included in this cluster also (Fig. 2). Species composition on the wet soil and wet rocks habitats was similar. More than half of the species are common on the sea marsh and alluvium on the riverside habitats.

The smallest width of ecological niche (NB $=0.08$ ) was typical for most species (37), e.g. Rivularia coadunata. Calothrix parietina $(\mathrm{NB}=$ 0.48), Chroococcus varius (0.38), Aphanocapsa muscicola (0.33) had the widest ecological amplitudes - these species had the greatest ecological flexibility in the studied habitats. We have pointed out earlier that Calothrix parietina shows wide ecological plasticity $(\mathrm{NB}=0.593)$ in

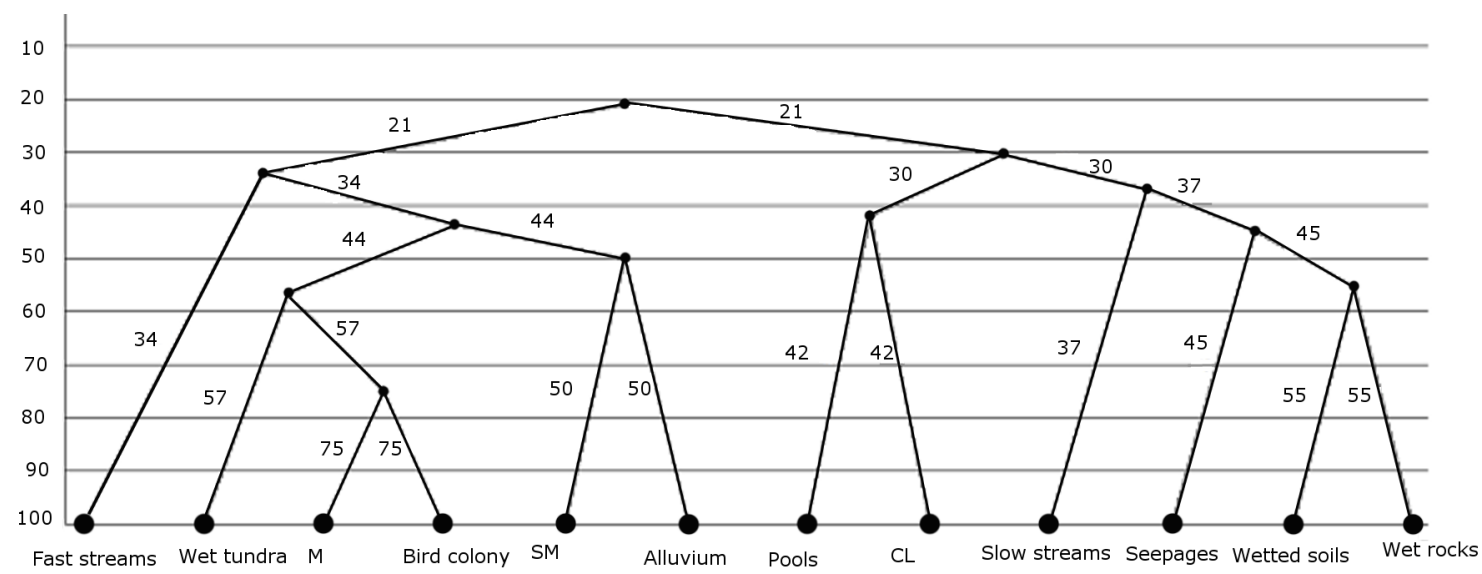

Fig. 2. Complete graph, based on Sørensen index, of floristic similarity of habitats; for clustering the mean distance (weighted pair-group method using arithmetic averaging) between elements of each cluster was used. Numbers show similarity in percent (scale from 0 to 100); M - meadow about cow-house, on the soil; CL - coast of the lake; SM - coastal of the sea, sea marsh. 
the Grønfjorden west coast (Davydov, 2011). Thus, the width of the ecological niche does not depend on the study area, and is an objective characteristic of this taxa.

Nostoc commune has, despite the high frequency of occurrence (in 23\% of samples) and wide distribution in the study area, a relatively narrow ecological niche $(\mathrm{NB}=0.27)$. This shows that a narrow optimum zone of species is on the different wet soils, but pessimum zone can be fairly wide (it can grow in streams and seepages).

Cyanobacterial floras in various territories of the Svalbard area differ from each other (Fig. 3). Most similar (the difference in species composition over $60 \%$ ) are the flora of the Grønfjorden west coast and flora of the Rijpfjorden east coast, however, only less than $1 / 3$ of species are common.

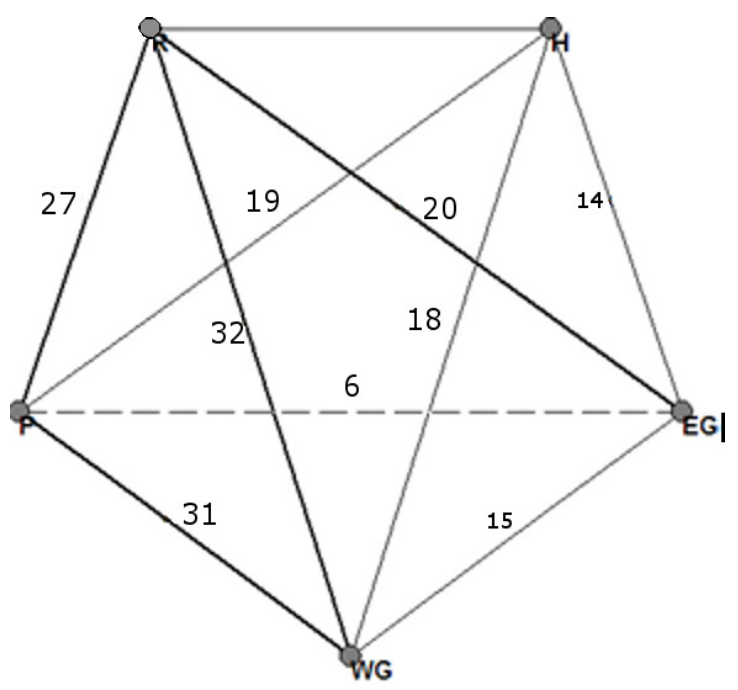

Fig. 3. Complete graph, based on Sørensen index, of floristic similarity between Svalbard areas; for clustering the mean distance (weighted pair-group method using arithmetic averaging) between elements of each cluster was used. Numbers show similarity in percent (scale from 0 to 100): P - flora of the vicinity settlement Pyramiden, R - flora of the Rijpfjorden east coast (Davydov, 2013), WG - flora of the Grønfjorden west coast (Davydov, 2011), EG - flora of the Grønfjorden east coast (Davydov, 2008), H - flora of the Revelva valley (Matuła et al., 2007).

\section{CONCLUSIONS}

A total of 73 cyanobacterial taxa were identified in the investigated area. Eleven species are first records for Spitsbergen flora. Cyanoprokaryotes in the vicinity of settlement Pyramiden were found in 12 habitat types. They were mostly confined to the hydro-terrestrial environments (Elster, 2002): wet slope seepages, wet soils, slow running tundra streams and pools. No species occured in all microhabitats. Nostoc commune (in $23 \%$ of samples) and Calothrix parietina (in $4.5 \%$ of samples) dominated in the most of habitats. Calothrix parietina, Chroococcus varius, Aphanocapsa muscicola had the widest ecological amplitude and the greatest ecological flexibility in the studied habitats.

\section{ACKNOWLEDGMENT}

This study was conducted with the support of grant from RFBR 14-04-98810.

\section{REFERENCES}

Davydov, D. A. 2005. Terrestrial cyanobacteria of east coast of Grønfjord (West Spitsbergen Island). Kompleksnye issledovaniya prirody Shpitsbergena [Complex investigations of Spitsbergen Nature] 5. (In Russian). Kola Science Centre RAS, Apatity, pp. 377-382.

Davydov, D. A. 2008. Cyanoprokaryota. In: Koroleva, N. E., Konstantinova, N. A., Belkina, O. A., Davydov, D. A. , Likhachev, A. Yu., Savchenko, A. N. \& Urbanavichiene, I. N. (eds): Flora and vegetation of Grønfjord area (Spitsbergen archipelago). K\&M, Apatity, pp. 93-102.

Davydov, D. A. 2010. Cyanoprokaryota of the Spitsbergen archipelago: the state of study. (In Russian). Botanicheskij zhurnal [Russian Botanical Journal] 95: 169-176.

Davydov, D. A. 2011. Diversity of the Cyanoprokaryota of the Grønfjord western coast (Spitsbergen, Svalbard). (In Russian). Botanicheskiy zhurnal [Russian Botanical Journal] 96: 1409-1420.

Davydov, D. 2013. Cyanoprokaryota in polar deserts of Rijpfjorden east coast, North-East Land (Nordaustlandet) Island, Spitsbergen. Algological Studies 142: 29-44. http:/ / dx.doi.org/10.1127/1864$1318 / 2013 / 0082$

Elster, J. 2002. Ecological classification of terrestrial algal communities in polar environments. In: Beyer, L. \& Bölter, M. (eds): Geoecology of Antarctic ice-free coastal landscapes. Springer Verlag, Berlin, Heidelberg, pp. 303-326. http://dx.doi. org/ 10.1007/978-3-642-56318-8_17 
Geitler, L. 1932. Cyanophyceae. In: Kolkwitz, R. (ed.): Rabenhorst's Kryptogamenflora von Deutschland, Österreich und der Schweiz 14. Akademischer Verlag, Leipzig. 1196 pp.

Hollerbach, M. M., Kosinskaja, E. K. \& Poljanskij, V. I. 1953. Sinezelenye vodorosli [Blue-green algae]. In: Opred. presnov. vodorosl. SSSR. 2. (In Russian). Izd. Sov. Nauka, Moskva. 652 pp.

Kaštovská, K., Elster, J., Stiball, M. \& Šantrůčková, H. 2005. Microbial Assemblages in Soil Microbial Succession after Glacial Retreat in Svalbard (High Arctic). Microbial Ecology 50: 396-407. http:// dx.doi.org/10.1007/s00248-005-0246-4

Kim, G. H., Klochkova, T. A. \& Kim, S. H. 2008. Notes on freshwater and terrestrial algae from Ny-Ålesund, Svalbard (high Arctic sea area). Journal of Environmental Biology 29(4): 485-491.

Kim, G. H., Klochkova, T. A., Han, J. W., Kang, S.H., Choi, H. G., Chung, K. W. \& Kim, S. J. 2011. Freshwater and Terrestrial Algae from Ny-Ålesund and Blomstrandhalvøya Island (Svalbard). Arctic 64(1): 25-21. http://dx.doi.org/10.14430/ $\operatorname{arctic} 4077$

Komárek, J. 2005. Cyanoprokaryota, 3. Teil: Heterocytous genera. In: Büdel, B., Gärtner, G., Krienitz, L. \& Schlager, M. (eds). Süsswasserflora von Mitteleuropa 19/3. Springer, Berlin Heidelberg, $1133 \mathrm{pp}$.

Komárek, J. \& Anagnostidis, K. 1998. Cyanoprokaryota, 1. Teil: Chroococcales. In: Ettl, H., Gärtner, G., Heynig, G. \& Mollenhauer, D. (eds). Süsswasserflora von Mitteleuropa 19/1. G. Fischer Verlag, Jena, 548 pp.

Komárek, J. \& Anagnostidis, K. 2005. Cyanoprokaryota, 1. Teil: Oscillatoriales. In: Büdel, B., Gärtner, G., Krienitz, L. \& Schlager, M. (eds). Süsswasserflora von Mitteleuropa 19/2. Elsevier, München, 759 pp.

Komárek, J., Kovacik, L., Elster, J. \& Komárek, O. 2012. Cyanobacterial diversity of Petuniabukta, Billefjorden, central Spitsbergen. Polish Polar Research 33: 347-368. http:/ /dx.doi.org/ 10.2478/ v10183-012-0024-1

Komárek, J., Taton, A., Sulek, J., Wilmotte, A., Kaštovská, K. \& Elster, J. 2006. Ultrastructure and taxonomic position of two species of the cyanobacterial genus Schizothrix. Cryptogamie, Algologie 27: 53-62.

Láska, K., Witoszová, D. \& Prošek, P. 2012. Weather patterns of the coastal zone of Petuniabukta, central Spitsbergen in the period 2008-2010. Polish Polar Research 33(4): 297-318. http:/ /dx.doi. org/ 10.2478/v10183-012-0025-0

Matuła, J. 1982. Investigations on the algal flora of West Spitsbergen. Acta Universitatis Wratislaviensis 525: 173-194.

Matuła, J., Pietryka, M., Richter, D. \& Wojtun, B. 2007. Cyanoprokaryota and algae of Arctic terrestrial ecosystems in the Hornsund area, Spitsbergen. Polish Polar Research 28: 283-315.
Matuła, J. \& Swies, F. 1989. Wstępna charakterystyka fykofl ory rejonu Bellsundu (Spitsbergen Zachodni) [Preliminary characteristics of algae in the south-western coast of Bellsund (Western Spitsbergen]. In: Polar Session. Natural Environment Research of West Spitsbergen. Maria CurieSklodowska University, Lublin, pp. 97-110.

Melechin, A. V., Davydov, D. A., Shalygin, S. S., Borovichev, E. A. 2013. Open information system on biodiversity cyanoprokaryotes and lichens CRIS (Cryptogamic Russian Information System). (In Russian). Bull. MOIP. Department of Biology 118(6): 51-56.

Nowakowskiy, A. B. 2004. Vozmozhnosti i principy programnogo modulya "GRAPHS" [Features and principles of the program module "GRAPHS"]. Institut biologii, Syktyvkar, 28 pp. (In Russian).

Oleksowicz, A. S. \& Luścińska, M. 1992. Occurrence of algae on tundra soils in Oscar II Land, Spitsbergen. Polish Polar Research 13: 131-147.

Perminova, G. N. 1990. Pochvennye vodorosli nekotoryh rajonov severa Evrasii i Dalnego Vostoka [Soil algae of some areas of northern Eurasia and the Far East]. (In Russian). Kirov, VINITI 4471-B90. 41 pp.

Petchurov, L. V. 1983. Spitsbergen. Moscow, 150 pp. Plichta, W. \& Luścińska, M. 1988. Blue-green algae and their infl uence on development of tundra soils in Kaffi öyra, Oscar II Land, Spitsbergen. Polish Polar Research 9: 475-484.

Richter, D., Matuła, J. \& Pietryka, M. 2009. Cyanobacteria and algae of selected tundra habitats in the Hornsund fiord area (West Spitsbergen). Oceanological and Hydrobiological Studies 38: 65-70.

Skulberg, O. M. 1996. Terrestrial and limnic algae and cyanobacteria. In: Elvebakk, A. \& Prestrud, P. (eds.). A catalogue of Svalbard plants, fungi, algae and cyanobacteria. Norsk Polarinstitutt, Skrifer, Oslo, pp. 383-395.

Stephenson, S. L. 1988. Distribution and ecology of myxomycetes in temperate forests. I. Patterns of occurrence in the upland forests of southwestern Virginia. Canadian Journal of Botany 66: 2187-2207.

Turicchia, S., Ventura, S., Schütte, U., Soldati, E., Zielke, M. \& Solheim B. 2005. Biodiversity of the cyanobacterial community in the foreland of the retreating glacier Midtre Lovènbreen, Spitsbergen, Svalbard. Algological Studies 117: 427-440. http://dx.doi.org/10.1127/18641318/2005/0117-0427

Willen, T. 1980. Phytoplankton from Lakes and Ponds on Vestspitsbergen. Acta Phytogeographica Suecica 68: 173-188.

Wolda, H. 1981. Similarity Indices, Sample Size and Diversity. Oecologia 50: 296-302. http://dx.doi. org/ 10.1007 / BF00344966 
24 Folia Cryptog. Estonica 